\title{
First Order Expectation Values of Electron Correlation Operators for Two-Electron Atoms
}

\author{
Boniface Otieno Ndinya ${ }^{1,2}$, Florence Mutonyi D'ujanga ${ }^{2}$, Jacob Olawo Oduogo ${ }^{3}$, \\ Andrew Odhiambo Oduor ${ }^{1}$, Joseph Omolo Akeyo ${ }^{1}$ \\ ${ }^{1}$ Department of Physics and Material Science, Maseno University, Maseno, Kenya \\ ${ }^{2}$ Department of Physics, Makerere University, Kampala, Uganda \\ ${ }^{3}$ Department of Physics, Masinde Muliro University of Science and Technology, Kakamega, Kenya \\ Email address: \\ bonifacendinya@gmail.com (B. O. Ndinya), ojakeyo04@yahoo.com (J. O. Akeyo),
}

\section{To cite this article:}

Boniface Otieno Ndinya, Florence Mutonyi D'ujanga, Jacob Olawo Oduogo, Andrew Odhiambo Oduor, Joseph Omolo Akeyo. First Order Expectation Values of Electron Correlation Operators for Two-Electron Atoms. American Journal of Modern Physics.

Vol. 4, No. 2, 2015, pp. 70-74.doi: 10.11648/j.ajmp.20150402.14

\begin{abstract}
Simple analytic first-order wave functions corresponding to two-electron atoms electron correlation operators are obtained by reduction of the Rayleigh-Schrödinger first order perturbation equation to that of one-electron through the partial integration over the variables of one electron. The resulting first order wave functions are applied to evaluate the first order expectation values of electron correlation operators associated with the radial correlation, magnetic shielding and diamagnetic susceptibility. The results obtained have close agreement with other theoretical results.
\end{abstract}

Keywords: First Order Wave Functions, Radial Correlation, Magnetic Shielding and Diamagnetic Susceptibility

\section{Introduction}

The application of quantum mechanics to the atomic problem results in a partial integro-differential equation [1]. This equation cannot be solved analytically except for the special case of the hydrogen atom. The solutions of the hydrogen atom provide useful insights regarding the nature of the atoms, but difficulties arise when we add one or more electrons. This is mainly because the strength of the electronelectron interactions is comparable to the nucleus-electron interaction [2-3]. Perturbation methods have been employed to calculate a wide range of atomic parameters relating to the ground states of the helium iso-electronic sequence, with the unperturbed eigenfunctions being products of screened hydrogenic orbital's [4-5]. The mean value associated to the magnetic shielding, diamagnetic susceptibility, relativistic mass corrections and dipole polarization were evaluated using first order wave functions obtained from a representation of the system by screened hydrogenic orbital. The perturbation theory of the helium iso-electronic sequence of the atoms has also been extended to obtain the first order density matrix equations, useful in evaluating the mean values of operators for the ground state of helium, helium ${ }^{3} \mathrm{~S}$ state and the lithium ground state [6]. Utpal R and Talukdar
B, [7], have studied the effects of electron-electron on the properties of helium iso-electronic sequence using variation approach and obtained the expectation value of the Hamiltonian and some radial operators. Sakho I., Ndao A S, Biaye $\mathrm{M}$ and Wague $\mathrm{A}$ [8], combined perturbation theory and the Ritz variation method, to obtain an analytic method called the screening constant by unit nuclear charge (SCUNC), to calculate of the ground-state energy, the first ionization energy and the radial correlation expectation value for the ground state of Helium like atoms. The SCUNC method has been extended in the calculation of the total energies, the total electron-electron interaction energies and the excitation energy for the doubly exited state of the Helium-like systems [9].

A simple analytic calculation for the first order wave function of helium-like system developed in a model in which nuclear charge screening is caused by repulsive coulomb interaction has been applied to calculate secondorder energies of the system within the model [10]. The firstorder wave function was obtained by reduction of the time independent first-order equation for the helium-like system to that of one-electron through partial integration over the variables of one electron. The resulting one-electron atoms equations, corresponding to the magnetic shielding term and 
the repulsive interactions term are the solved analytically. The total energy to second order correction were found to have close agreement with those from the Hatree-Fock model using arbitrary first-order wave functions.

In the paper the first order expectation values of the twoelectron atoms electron correlation operators associated with the radial correlation, magnetic shielding and diamagnetic susceptibility are evaluated using first order wave functions corresponding to each operator. The first order wave functions associated to each operators is obtained by solving the first order time independent Schrödinger equation for the helium-like system analytically, using a model in which nuclear charge screening is caused by the repulsive coulomb interaction.

\section{General Dynamics and Perturbation Theory of the System}

The Hamiltonian governing the dynamics of the two electrons in the model in which nuclear charge screening is caused by the repulsive coulomb interaction takes the form (atomic units) [11]

$$
H=H^{(0)}+H^{\prime}
$$

where the unperturbed Hamiltonian is

$$
H^{(0)}=-\frac{1}{2} \nabla_{1}^{2}-\frac{1}{2} \nabla_{2}^{2}-\frac{\zeta}{r_{1}}-\frac{\zeta}{r_{2}}
$$

and the perturbing Hamiltonian is

$$
H^{\prime}=-\frac{1}{4}\left(\frac{1}{r_{1}}+\frac{1}{r_{2}}\right)+\frac{1}{2}\left(\frac{S_{12}}{r_{1}}+\frac{S_{21}}{r_{2}}\right)
$$

The effective nuclear charge $\zeta$ in $(1 b)$ is defined as

$$
\zeta=Z-\frac{1}{4}
$$

and the nuclear charge screening parameter $S_{i j}, i, j=1,2$ in (1c) is expressed as

$$
S_{i j}=\left(1+\left(\frac{r_{j}}{r_{i}}\right)^{2}-2\left(\frac{r_{j}}{r_{i}}\right) \cos \vartheta\right)^{-1 / 2}, \quad r_{i} \geq r_{j}
$$

where $r_{i}=\left|\mathbf{r}_{i}\right|, \quad r_{j}=\left|\mathbf{r}_{j}\right|$ and $\vartheta$ is the orientation angle between the position vectors $\mathbf{r}_{i}$ and $\mathbf{r}_{j}$ of the electron pair $(i, j)$.The unperturbed eigenvalue equation is

$$
H^{(0)} \Psi^{(0)}=E^{(0)} \Psi^{(0)}
$$

where the zero-order wave function is

$$
\Psi^{(0)}=\frac{\zeta^{3}}{\pi} \exp \left(-\zeta\left(r_{1}+r_{2}\right)\right)
$$

and energy value is [11]

$$
E^{(0)}=-\zeta^{2}=-\left(Z-\frac{1}{4}\right)^{2}
$$

The first order approximation of the Rayleigh-Schrödinger perturbation theory procedure leads to a homogeneous equation

$$
\left(H^{(0)}-E^{(0)}\right) \Psi^{(1)}+\left(H^{\prime}-E^{(1)}\right) \Psi^{(0)}=0
$$

The knowledge of the form of the zero-order wave function $\Psi^{(0)}$ and first order wave function $\Psi^{(1)}$ obtained in (4a) leads to the values of the first and second order corrections to the energy, expressed as

$$
E^{(1)}=\left\langle\Psi^{(0)}\left|H^{\prime}\right| \Psi^{(0)}\right\rangle,
$$

and

$$
E^{(2)}=\left\langle\Psi^{(0)}\left|H^{\prime}-E^{(1)}\right| \Psi^{(1)}\right\rangle
$$

respectively $[1,12]$.

The expectation value of an operator $\mathbf{L}_{k}$ of the system may be expanded into different orders $[4,5]$

$$
\left\langle\mathbf{L}_{k}\right\rangle=L_{k}^{(0)}+L_{k}^{(1)}+\ldots \ldots \ldots
$$

where

$$
L_{k}^{(0)}=\left\langle\Psi_{k}^{(0)}\left|\mathbf{L}_{k}\right| \Psi_{k}^{(0)}\right\rangle
$$

is the zero-order approximation to the diagonal matrix element of the dynamical variable and

$$
L_{k}^{(1)}=\left\langle\Psi_{k}^{(0)}\left|\mathbf{L}-L_{k}^{(0)}\right| \chi_{k}^{(1)}\right\rangle
$$

is the first order approximation. The first order wave function $\chi_{k}^{(1)}$ of the system satisfies the equation

$$
\left(H^{(0)}-E_{k}^{(0)}\right) \chi_{k}^{(1)}+\left(\mathbf{L}-L_{k}^{(0)}\right) \Psi_{k}^{(0)}=0
$$

Since $H^{(0)}, E_{k}^{(0)}, \Psi_{k}^{(0)}, \mathbf{L}$ and $L_{k}^{(0)}$ are known, the solution of (5d) yield the value of $\chi_{k}^{(1)}$, that is used to evaluate $L_{k}^{(1)}$ in $(5 \mathrm{c})$.

\subsection{Radial Correlation}

The radial correlation of the Helium like atoms system within the model in which nuclear charge screening is caused by the repulsive coulomb interaction, is defined from (1c) by [11]

$$
\mathbf{L}_{\text {rad }}=\frac{1}{\mathbf{r}_{12}}=\frac{1}{2}\left(\frac{S_{12}}{r_{1}}+\frac{S_{21}}{r_{2}}\right)
$$

Substituting (6a) in (5b), taking $\Psi_{k}^{(0)}=\Psi^{(0)}$ and evaluating the resulting expression, the zero-order expectation of the 
radial correlation term becomes

$$
L_{\text {rad }}^{(0)}=\left\langle\Psi^{(0)}\left|\mathbf{L}_{\text {rad }}\right| \Psi^{(0)}\right\rangle=\frac{5}{8} \zeta
$$

The first order perturbation equation ( $5 \mathrm{~d}$ ) cannot be solved directly for $\chi_{\text {rad }}^{(1)}$, since the radial correlation term contains two electron terms. However, analytic solutions to $(5 \mathrm{~d})$ can be found if $\chi_{\text {rad }}^{(1)}$ corresponds to a one-electron operator $\mathbf{L}_{\text {rad }}$.

Following the Rayleigh-Schrodinger $1 / \zeta$ perturbation expansion, the two-electron atoms equation is reduced to that of one electron through partial integration over the variables of one electron [10]. The resulting first-order wave function is

$$
\chi_{\text {rad }}^{(1)}=\left(f\left(r_{1}\right)+f\left(r_{2}\right)\right) \psi_{s c}^{(0)}
$$

where

$$
\begin{aligned}
f\left(r_{i}\right)= & -\frac{23}{32}-\frac{1}{4} \exp \left(-2 r_{i}\right)-\frac{3}{8} \log \left(r_{i}\right) \\
& -\frac{3}{8} \Gamma+\frac{3}{16 r_{i}}\left(1-\exp \left(-2 r_{i}\right)\right) \\
& +\frac{5}{8} r_{i}+\frac{3}{8} \exp \text { Integral } E_{i}\left(-2 r_{i}\right)
\end{aligned}
$$

and

$$
\psi_{s c}^{(0)}=\frac{1}{\pi} \exp \left(-\left(r_{1}+r_{2}\right)\right)
$$

is the scaled zero order wave function (3b). Using equation (6b), the scaled zero order expectation value is $L_{r a d_{s c}}^{(0)}=5 / 8$.
The first order expectation value of the radial correlation term $(5 \mathrm{c})$, becomes

$$
L_{\text {rad }}^{(1)}=\left\langle\psi_{s c}^{(0)}\left|\mathbf{L}-L_{\text {rad }_{s c}}^{(0)}\right| \chi_{\text {rad }}^{(1)}\right\rangle=-0.111003 a . u,
$$

after substituting for (6a), (7b) and (7c). Using (6b) and (8a) in $(5 a)$, the expectation value of the radial correlation operator to first order approximation is

$$
\begin{aligned}
\left\langle\mathbf{r}_{12}^{-1}\right\rangle_{p} & =L_{r a d}^{(0)}+L_{r a d}^{(1)}=\frac{5}{8} \zeta-0.111003 \\
& =\frac{5}{8}\left(Z-\frac{1}{4}\right)-0.111003
\end{aligned}
$$

Table 2.1 shows the calculated values of the radial correlation expectation value $\left\langle\mathbf{r}_{12}\right\rangle_{p}^{-1}(8 \mathrm{~b})$ for $\mathrm{Z}=1-6$, compared to theoretical results obtained in Utpal $\mathrm{R}$ and Talukdar B [7] andSakho I. et al, 2006 [8]. In spite of the choice of simple wave functions which depend only on the distance coordinates $r_{1}$ and $r_{2}$ the theoretical results for the radial correlation term $\left\langle\mathbf{r}_{12}^{-1}\right\rangle_{p}$ have a close agreement with those of

\begin{tabular}{|c|c|c|c|c|c|c|}
\hline & $H^{-}$ & $\mathrm{He}$ & $\mathrm{Li}^{+}$ & $B e^{2+}$ & $B^{3+}$ & $C^{4+}$ \\
\hline$\left\langle\mathbf{r}_{12}^{-1}\right\rangle_{\text {Sakho }}$ & 0.4297 & 1.0547 & 1.6797 & 2.3047 & 2.9297 & 3.5547 \\
\hline$\left\langle\mathbf{r}_{12}^{-1}\right\rangle_{U t p a l}$ & 0.3744 & 0.9820 & 1.6010 & 2.2233 & 2.8463 & 3.4700 \\
\hline$\left\langle\mathbf{r}_{12}^{-1}\right\rangle_{p}$ & 0.357747 & 0.982747 & 1.60775 & 2.23275 & 2.85775 & 3.48275 \\
\hline
\end{tabular}
Utpal and Talukdar, [7] who constructed in their analytical model a wave function depending on the position coordinates $s=r_{1}+r_{2}$ and $r_{12}$. The theoretical results are better than those obtained by Sakho, et al, 2006[8] using SCUNC method. The results also compares favourbly with exact values for radial correlation expectation value for $H e=0.9458$ a.u and $C^{4+}=3.4389$ a.u quaoted from the variational calculation of Pekeris [13].

\subsection{Magnetic Shielding}

Within the model in which nuclear charge screening is caused by the repulsive coulomb interaction the magnetic shielding operator $\mathbf{L}_{m a g}$, is defined from (1c) as

$$
\mathbf{L}_{\text {mag }}=\left(r_{1}^{-1}+r_{2}^{-1}\right)
$$

Substitute (9a) in (5b), the zero-order expectation of the magnetic shielding term becomes

$$
L_{\text {mag }}^{(0)}=\left\langle\Psi^{(0)}\left|\mathbf{L}_{\text {mag }}\right| \Psi^{(0)}\right\rangle=2 \zeta
$$

The magnetic shielding term (9a) contains one electron terms, therefore the first order perturbation equation (5d) can be solved directly for variables of one electron, to obtain

$$
\chi_{m a g}^{(1)}=\left(r_{1}+r_{2}\right) \Psi^{(0)}
$$

The first order correction to the expectation value of the magnetic shielding term $(5 \mathrm{c})$ is

$$
L_{\text {mag }}^{(1)}=2\left\langle\Psi^{(0)}\left|\mathbf{L}-L_{\text {mag }}^{(1)}\right| \chi_{\text {mag }}^{(1)}\right\rangle=-0.125 \text { a.u, }
$$

Using (9b) and (10a) in (5a), the expectation value of the magnetic shielding term to first order approximation is 


$$
\begin{aligned}
\left\langle\left(r_{1}^{-1}+r_{2}^{-1}\right)\right\rangle_{p} & =L_{\text {mag }}^{(0)}+L_{\text {mag }}^{(1)}=2 \zeta-0.1250 \\
& =2(Z-1 / 4)-0.1250
\end{aligned}
$$

Table 2.2 shows the calculated values of the one electron radial correlation term associated with magnetic shielding for $1 / 2\left\langle r_{1}^{-1}+r_{2}^{-1}\right\rangle_{p}(10 \mathrm{~b})$ for $\mathrm{Z}=1-10$

\subsection{Diamagnetic Susceptibility}

The diamagnetic susceptibility is proportional to the expectation value of

$$
\mathbf{L}_{d i a}=r_{1}^{2}+r_{2}^{2}
$$

The zero order expectation value is

$$
L_{\text {dia }}^{(0)}=\left\langle\Psi^{(0)}\left|r_{1}^{2}+r_{2}^{2}\right| \Psi^{(0)}\right\rangle=6 / \zeta^{2}
$$

Substitute (11a) and (11b) in (5d) and use steps in section 2.2.2, the first order wave function become

$$
\chi_{\text {dia }}^{(1)}=-\left(\frac{r_{1}^{2}+r_{2}^{2}}{\zeta^{2}}+\frac{r_{1}^{3}+r_{2}^{3}}{3 \zeta}\right) \Psi^{(0)}
$$

Substitute (11a), (11b) and (11c) in (5c), the first order correction to the expectation value of the diamagnetic susceptibility term, becomes

$$
L_{\text {dia }}^{(1)}=\left\langle\Psi^{(0)}\left|\mathbf{L}-L_{\text {dia }}^{(0)}\right| \chi_{\text {dia }}^{(1)}\right\rangle=-129 / 32 \zeta^{6}
$$

and the corresponding first order expectation value

$$
\left\langle r_{1}^{2}+r_{2}^{2}\right\rangle_{p}=L_{\text {dia }}^{(0)}+L_{\text {dia }}^{(1)}=6 / \zeta^{2}-129 / 32 \zeta^{6}
$$

Table 2.2 shows the calculated values of the one electron radial correlation term associated with magnetic shielding for $1 / 2\left\langle r_{1}^{2}+r_{2}^{2}\right\rangle_{p}(12 b)$ for $\mathrm{Z}=1-10$.

\subsection{The Radial Operator $\left(r_{1}+r_{2}\right)$}

Using steps in equations (11a) - (12c), it is easy to show that the first order wave function associated with the radial operator $\left(r_{1}+r_{2}\right)$ is

$$
\chi^{(1)}=-\frac{1}{2}\left(r_{1}^{2}+r_{2}^{2}\right) \Psi^{(0)}
$$

\begin{tabular}{|c|c|c|c|c|c|c|}
\hline Atom & $1 / 2\left\langle r_{1}^{-1}+r_{2}^{-1}\right\rangle_{p}$ & $\left\langle r_{1}^{-1}\right\rangle_{\text {Utpal }}$ & $1 / 2\left\langle r_{1}+r_{2}\right\rangle_{p}$ & $\langle r\rangle_{\text {Utpal }}$ & $1 / 2\left\langle r_{1}^{2}+r_{2}^{2}\right\rangle_{p}$ & $\left\langle r^{2}\right\rangle_{\text {utpal }}$ \\
\hline$H^{-}$ & $\begin{array}{l}0.6875 \\
0.6883^{\mathrm{a}}\end{array}$ & 0.6938 & 1.7778 & 2.1800 & 5.9918 & 6.3222 \\
\hline $\mathrm{He}$ & $\begin{array}{l}1.6875 \\
1.6683^{\mathrm{a}}\end{array}$ & 1.6888 & $\begin{array}{l}0.8397 \\
0.9295^{\mathrm{a}}\end{array}$ & 0.8907 & $\begin{array}{l}0.9094 \\
1.1935^{\mathrm{a}}\end{array}$ & 1.0558 \\
\hline$L i^{+}$ & $\begin{array}{l}2.6875 \\
2.6879^{\mathrm{a}}\end{array}$ & 2.6879 & 0.5410 & 0.5591 & 0.3920 & 0.4161 \\
\hline$B e^{2+}$ & 3.6875 & 3.6880 & 0.3982 & 0.4073 & 0.2126 & 0.2209 \\
\hline$B^{3+}$ & 4.6875 & 4.6876 & 0.3149 & 0.3203 & 0.1328 & 0.1367 \\
\hline$C^{4+}$ & $\begin{array}{l}5.6875 \\
5.6876^{\mathrm{a}}\end{array}$ & 5.6875 & $\begin{array}{l}0.2604 \\
0.2668^{\mathrm{a}}\end{array}$ & 0.2640 & $\begin{array}{l}0.0907 \\
0.0957^{\mathrm{a}}\end{array}$ & 0.0928 \\
\hline$N^{5+}$ & 6.6875 & 6.6876 & 0.2219 & 0.2245 & 0.0658 & 0.0671 \\
\hline$O^{6+}$ & 7.6875 & 7.6877 & 0.1934 & 0.1952 & 0.0499 & 0.0508 \\
\hline$F^{7+}$ & 8.6875 & 8.6875 & 0.1713 & 0.1728 & 0.0392 & 0.0398 \\
\hline$N e^{8+}$ & $\begin{array}{l}9.6875 \\
9,6875^{\mathrm{a}}\end{array}$ & 9.6877 & $\begin{array}{l}0.1537 \\
0.1559^{\mathrm{a}}\end{array}$ & 0.1549 & $\begin{array}{l}0.0316 \\
0.0326\end{array}$ & 0.0320 \\
\hline
\end{tabular}

and the first order expectation value is

$$
\begin{aligned}
\left\langle r_{1}+r_{2}\right\rangle_{p} & =L^{(0)}+L^{(1)}=3 / \zeta-3 / 16 \zeta^{3} \\
& =3 /(Z-1 / 4)-3 / 16(Z-1 / 4)^{3}
\end{aligned}
$$

Table 2.2 shows the calculated expectation values for the one electron radial operator $1 / 2\left\langle r_{1}+r_{2}\right\rangle_{p}(13 b)$ for $\mathrm{Z}=1-8$.

Table 2.2. Theoretical values of the one-electron radial correlation term associated with $1 / 2\left\langle r_{1}^{-1}+r_{2}^{-1}\right\rangle_{p}(10 b), 1 / 2\left\langle r_{1}+r_{2}\right\rangle_{p}(13 b)$ and $1 / 2\left\langle r_{1}^{2}+r_{2}^{2}\right\rangle_{p}(12 b)$ for $Z=1-10$, compared to theoretical results obtained in Utpal $R$ and Talukdar B, 1999 [7] and exact value (marked a) obtained in Pekeris, 1958 [13].

In table 2.2 the theoretical results for the first order expectation value for $1 / 2\left\langle r_{1}^{-1}+r_{2}^{-1}\right\rangle_{p}$ is close with those obtained by Utpal R and Talukdar B, 1999 [9] for one electron radial correlation term $\left\langle r_{i}^{-1}\right\rangle$ and the exact values obtained by Pekeris, 1958 [13] using variation method. The theoretical result for first order expectation value for $1 / 2\left\langle r_{1}+r_{2}\right\rangle_{p}$ has close agreement with the expectation value obtained by UtpalR and Talukdar, 1999 [9] for one electron radial correlation term $\left\langle r_{i}\right\rangle$ and exact values by Pekeris, 1958 [13] for $Z \geq 3$. Similarly the theoretical value for $1 / 2\left\langle r_{1}^{2}+r_{2}^{2}\right\rangle_{p}$ has close agreement with the expectation value obtained by Utpal R and Talukdar B, 1999 [7] for one electron radial correlation term $\left\langle r_{i}^{2}\right\rangle$ and exact values by Pekeris, 1958 [13] for $Z \geq 3$. The slight disparities in the values of expectation 
values of $1 / 2\left\langle r_{1}+r_{2}\right\rangle_{p}$ and $1 / 2\left\langle r_{1}^{2}+r_{2}^{2}\right\rangle_{p}$ for $H^{-}$and $H e$ may be attributed the choice of screening constant which underestimate the zero order expectation value and choice of simple wave function which depend on only the distance coordinate $s=r_{1}+r_{2}$,

\section{Conclusion}

In section 2.1 , the theoretical results for the expectation value to the radial correlation term is found using a first order wave function order wave function obtained by following a simple analytic method. The analytic method involves applying the Rayleigh-Schrodinger perturbation expansion to the two-electron atom and reducing the resulting equation to that of one-electron through partial integration over the variables of one electron. The resulting first order wave function $(7 a)$ is used to calculate first order correction to the zero order expectation value of the radial correlation term (8b). In table 2.1, the theoretical results for the radial correlation term obtained using (8b) have a close agreement with other theoretical results.

In section 2.2, 2.3 and 2.4 the first order wave functions associated with the magnetic shielding term $\left(r_{1}^{-1}+r_{2}^{-1}\right)$, diamagnetic susceptibility term $\left(r_{1}^{2}+r_{2}^{2}\right)$ radial operator $\left(r_{1}+r_{2}\right)$ of the helium like atoms, given by (9c), (11c) and (13a), were obtained directly by solving (5d), since they contain only one electron terms. The wave functions were used to calculate the first order corrections to the zero order expectation values of the radial operators $\left\langle r_{1}^{-1}+r_{2}^{-1}\right\rangle(10 \mathrm{~b})$, $\left\langle r_{1}^{2}+r_{2}^{2}\right\rangle(12 b)$ and $\left\langle r_{1}+r_{2}\right\rangle$ (13b). Since the wave function is a symmetric function of $r_{1}$ and $r_{2}$ and the values for $r_{1}$ and $r_{2}$ are the same, then (10b), (12b) and (13b) can be applied to obtain the one-electron radial expectation value as given in table 2.2. The one-electron radial correlation values have close agreement with other theoretical result for $Z \geq 3$.

\section{Acknowledgement}

Boniface Ndinya thanks DAAD for the staff exchange in Sub-Saharan Africa fellowship code number A/14/93695 at
Makerere University, Makerere University for office space and hospitality and Maseno University for the permission to be away.

\section{References}

[1] Bethe H A and Salpeter E E. (1957). Quantum mechanics of one- and two-electron Atoms. Berlin: Springer-Verlag.

[2] Coundon E U and Shortely G H. (1959). The theory of atomic spectra. London: Camdridge University Press.

[3] Greiner, W. (2000). Quantum Mechanics. Berlin: Springer Verlag.

[4] Dalgarno A and Stewart A L. (1958). A perturbation calculation for properties of helium iso-electronic sequence. Proc. Roy. Soc.( London), A 247, 245-249.

[5] Dalgarno A and Stewart A L. (1960). The screening approximation for the Helium Sequence. Proc. Roy. Soc. A, 534-540.

[6] Hall G G, Jones L L and Rees D. (1965). The first-order density matrix for the direct calculation of atoms. Proc. Roy. Soc. A , 194-202

[7] Utpal R and Talekdar B. (1999). Electron correction for Helium-like stoms. Physica Scripta, Vol 59, 133-137.

[8] Sakho I. Ndao A S, Biaye M and Wague A. (2006). Calculation of the groung state energy, the first ionization energy and radial correlation expectation value for He-like atoms. Phy, Scr. 74, 180-18

[9] Sakho I, Ndao A S, Biaye M and Wague A. (2008). Screening constant by unit nuclear charge for (ns)S, (np)D and (nsnp) $\mathrm{P}$ excited states of He-like system. Eur. Phys. J. D 47, 37-44.

[10] Ndinya B O and Omolo J A. (2010). A direct calculation of first order wave function of Helium. Commun. Thor. Physics. $54,647-650$.

[11] Omolo J A and Ndinya B O. (2009). Repulsive Coulomb interaction and nuclear charge screening in Helium and Helium-like ions. Indian. J. Theor. Phys. 58, 81-85.

[12] Merzbacher, E. (1970). Quantum Mechanics. New York: John Wiley and son.

[13] Pekeris, C. (1958). Ground state of two electron atoms. Phys. Rev. $112,1649$. 